\title{
Delirium in Frail Older Adults
}

\author{
Min Ji Kwak \\ Joan and Stanford Alexander Division of Geriatric and Palliative Medicine, McGovern Medical School, The University of Texas Health Science Center at Houston, \\ Houston, TX, USA
}

Corresponding Author:

Min Ji Kwak, MD, MS, DrPH

Joan and Stanford Alexander Division

of Geriatric and Palliative Medicine,

McGovern Medical School, The

University of Texas Health Science

Center at Houston, 6431 Fannin St

MSB 5.126, Houston, TX 77030, USA

E-mail:min.ji.kwak@uth.tmc.edu

ORCID:

https://orcid.org/0000-0003-2778-3984

Received: July 27, 2021

Revised: August 27, 2021

Accepted: August 27, 2021
Delirium and frailty are prevalent geriatric syndromes and important public health issues among older adults. The prevalence of delirium among hospitalized older adults ranges from 15\% to $75 \%$, while that of frailty ranges from $12 \%$ to $24 \%$. The exact pathophysiology of these two conditions has not been clearly identified, although several hypotheses have been proposed. However, these conditions are considered to be multifactorial in etiology and are associated with inflammation related to aging, alterations in vascular systems, genetics, and nutritional deficiency. Furthermore, clinically, they are significantly associated with each other, for example, frailty increases the risk of delirium almost two- to three-fold among hospitalized older adults. With their multifactorial etiology and unknown pathophysiology, current evidence supports more practical multicomponent patient-centered approaches to prevent and manage delirium among hospitalized older adults. These comprehensive and organized bundled approaches can identify highrisk patients with frailty and more effectively manage their delirium.

Key Words: Frailty, Delirium, Geriatric assessment

\section{INTRODUCTION}

Delirium and frailty are both important geriatric syndromes that commonly occur among older adults. Delirium is an acute medical condition characterized by changes in mental and cognitive status and usually occurs among hospitalized older adults, ${ }^{1)}$ with a prevalence ranging from $15 \%$ to $75 \%$, depending on the clinical setting. Frailty is a chronic condition characterized by decreased functional status and increased vulnerability to various stressors, with a prevalence ranging from $12 \%$ to $24 \% .^{3-5)}$ Although they are different conditions, delirium and frailty also share commonalities and have overlapping features. This study reviewed the definitions and pathophysiologies of these two geriatric conditions, described their similarities and differences, and provided current evidence of delirium management among older adults with frailty.

\section{DELIRIUM}

\section{Epidemiology of Delirium}

Delirium is a common geriatric syndrome and is defined as an acute and fluctuating change in mental and cognitive status, which frequently occurs in hospitalized older adults. It is characterized by impaired attention, reduced awareness of surroundings, disorientation, abnormal arousal, behavioral abnormalities, and disturbance in visuospatial perception. ${ }^{1)}$ A recent systematic review reported an overall delirium prevalence of $23 \%$ (95\% confidence interval [CI], 19\%-26\%), although there was some degree of variation depending on the diagnostic criteria used. ${ }^{6)}$ The prevalence may be higher among surgical patients, at 15\%-25\% after major elective surgery and 50\% after high-risk procedures such as hip fracture or cardiac surgery. ${ }^{2)}$ Patients in the intensive care unit more often experience delirium, with an overall prevalence ranging from $31 \%$ to $75 \% .{ }^{2,7)}$ In addition to high prevalence, delirium is a serious public 
health problem that is associated with poor clinical outcomes. Delirium is independently associated with a two- to four-fold increased risk of mortality, a pattern that has been mostly consistent across various clinical settings and illnesses. ${ }^{8-16)}$ The additional adverse outcomes of delirium in older adults include prolonged hospitalization, higher chances of discharge to nursing homes, declines in cognitive function, and increased healthcare costs. ${ }^{2,8,13,17-19)}$

\section{Pathophysiology of Delirium}

While the pathophysiology of delirium remains unclear, its etiology is widely accepted to be multifactorial. Delirium was previously considered to be a disorder of neurotransmitters such as acetylcholine, melatonin, dopamine, norepinephrine, glutamate, 5-hydroxytryptamine, serotonin, histamine, and/or gamma-aminobutyric acid. $^{20)}$ However, more recent theories suggest that the pathophysiology of delirium is more complex. For example, delirium may result from a combination of neurotransmitter disorders coupled with a failure in processing sensory signals or motor effectors and a breakdown in the brain network. Furthermore, such conditions may interact with neuroinflammation, excess oxidative stress, neuroendocrine dysfunction, circadian rhythm, or melatonin dysregulation. ${ }^{20)}$ This complex pathophysiology of delirium becomes more complicated in patients with acute medical illnesses. Acute illnesses such as sepsis or pneumonia can cause brain hypoperfusion and impair glucose supply, subsequently leading to insufficiency of brain bioenergetics and breakdown of the brain network. ${ }^{21,22)}$ However, the alterations in body composition in older adults, including a reduction in lean mass and an increase in fat mass, could also play an important role in the development of delirium by adding more inflammatory stimuli via the endocrine secretion of pro-inflammatory adipokines. ${ }^{23)}$

\section{FRAILTY}

\section{Epidemiology of Frailty}

Frailty is a clinical condition characterized by a progressive decline in homeostatic capacity and increased vulnerability to endogenous and exogenous stressors. ${ }^{5,2425)}$ Frailty is prevalent among older adults, and along with the growing aging population, it is an important public health issue worldwide. ${ }^{4)}$ Although it varies across studies and diagnostic tools, the prevalence of frailty worldwide ranges from $8 \%$ to $24 \% .{ }^{3,4)}$ In America, the prevalence of frailty based on physical phenotype and deficit accumulation models was $17 \%$ and $23 \%$, respectively. ${ }^{4}$ Furthermore, as the consequence of increased vulnerability to various stressors, frailty is associated with an increased risk for adverse health outcomes, including a decline in functional status, prolonged length of stay in the hospital, increased risk of institutionalization, and higher mortality, ${ }^{5,24,25)}$

\section{Pathophysiology of Frailty}

Like delirium, the pathophysiology of frailty has not been completely elucidated. Furthermore, despite its significance in public health and its association with negative health outcomes, there is no internationally agreed definition for frailty. Two models are widely used to assess frailty: the frailty phenotype ${ }^{26)}$ and the deficit accumulation frailty index. ${ }^{5,27-29)}$ The frailty phenotype views frailty as a condition with certain clinical characteristics based on the presence or absence of specific physical conditions such as weight loss, exhaustion, weakness, low walking speed, and decreased physical activity. The deficit accumulation frailty index assesses frailty using the scale of the total burden of age-related health deficits in older adults, including symptoms, signs, diseases, and disabilities. ${ }^{30)}$

Regardless of approach, age-related changes in multiple physiological systems or organs with certain characteristics are the foundation of the development of frailty. Among physiologic changes in the aging body, alterations in the neuromuscular, neuroendocrine, and immunological systems play important roles. ${ }^{31)}$ Furthermore, changes in each system interact cumulatively, leading to an overall decline in function, eventually reaching the threshold beyond which older adults' capacity to sustain minor stress is compromised. $^{31}$

\section{SIMILARITIES AND DIFFERENCES BETWEEN DELIRIUM AND FRAILTY}

As both delirium and frailty result from the disintegration of balance and homeostasis across multiple body systems, they are intuitively related and share several commonalities. They occur commonly among older adults, are multifactorial in their etiology, and are associated with poor clinical outcomes, including increased risk of functional loss, institutionalization, and mortality. ${ }^{32)}$ The exact pathophysiologies of these conditions have not yet been identified, although they share common biologic processes, including inflammation, alterations in vascular systems, genetics, and nutritional deficiency in their development (Fig. 1). ${ }^{33}$ As individuals age, their levels of circulating inflammatory cytokines increase, a condition that may be heightened in frailty. With higher baseline cytokine levels, the pro-inflammatory response to stressors becomes more prominent, making individuals more susceptible to stressors and explaining the development of both delirium and frailty. ${ }^{34,35)}$ Additionally, alterations in the vascular system, particularly small vessel disease, have been associated with geriatric syndromes. As common geriatric syndromes, delirium and frailty are 


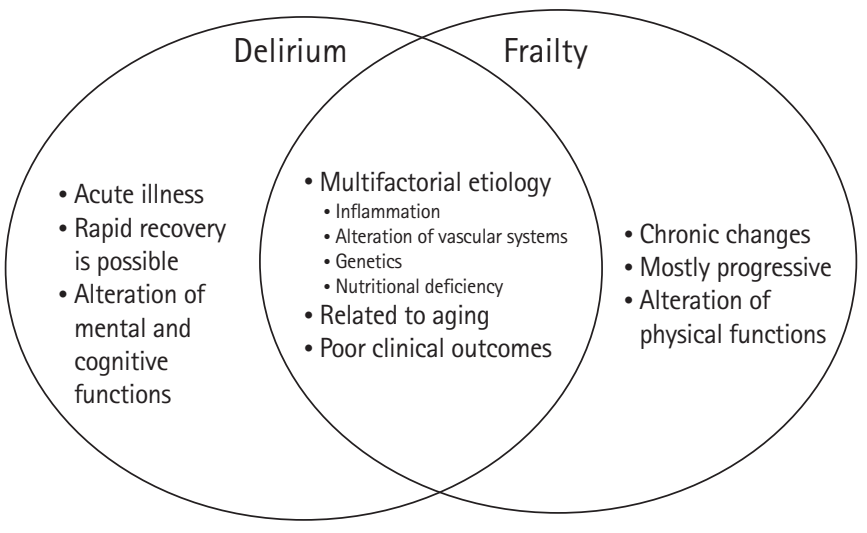

Fig. 1. Similarities and differences between delirium and frailty.

also likely to be associated with small vessel diseases. ${ }^{36)}$ Moreover, frailty is a subclinical marker of cardiovascular disease, and delirium occurs twice as often among individuals undergoing cardiovascular surgery compared with that among patients undergoing orthopedic or abdominal surgeries. ${ }^{37-39)}$ Malnutrition, reduction in caloric intake, and subsequent weight loss are prevalent in older adults and may lead to sarcopenia and thus frailty. ${ }^{40)}$ According to the phenotype frailty model, weight loss is a prominent characteristic of frailty. ${ }^{26)}$ Furthermore, malnutrition is considered an important predisposing risk factor for delirium. ${ }^{41)}$

Although frailty and delirium share common features, as described above, there are also differences between the two conditions (Fig. 1). Frailty results from a long-term decline in functions across an individual's multiple systems, and its process is considered chronic and progressive. In contrast, delirium is an acute process or reaction to a newly introduced stressor. Delirium may progress and resolve rapidly compared to frailty, although full recovery could take weeks or even months. ${ }^{23)}$ Delirium is mainly characterized by alterations in mental and cognitive functions, while frailty is more often characterized by changes in physical function and the accumulation of physical deficits. When multiple insults affect an already susceptible individual, the brain may not compensate, leading to delirium.

\section{DELIRIUM IN FRAIL OLDER ADULTS}

Therefore, despite their differences, from a practical perspective, frailty and delirium show important overlapping pictures: individuals already vulnerable to various stressors, e.g., older adults with pre-existing frailty, are at a greater risk for delirium. Several studies on the association between delirium and frailty in older adults showed that frailty was associated with an increased risk of delirium compared to those who do not have frailty. In 2018, a system- atic review and meta-analysis of 999 patients by Presico et al. ${ }^{42}$ showed a 2.19-fold increased risk of delirium among frail patients (risk ratio $[\mathrm{RR}]=2.19 ; 95 \% \mathrm{CI}, 1.65-2.91$ ). Although limited to surgical patients, a meta-analysis of the cohort study by Fu et al. ${ }^{43)}$ reported that frailty patients had an increased risk for delirium, with an odds ratio (OR) of 3.23 (95\% CI, 2.56-4.07). More recently, in 2021, Zhang et al. .4) $^{43}$ reported a meta-analysis study including 217,623 patients and showed that frailty was associated with an increased risk for delirium compared to the risk in patients without frailty ( $\mathrm{OR}=2.96$; 95\% CI. 2.36-3.71). They also conducted further analyses to assess the association in different patient groups. Frailty was associated with a 2.43 -fold increased risk of delirium (95\% CI, 1.88-3.14) in selective surgical patients, a 3.61fold increased risk (95\% CI, 1.65-7.89) in medical patients, a 3.76fold increased risk (95\% CI, 2.88-4.92) in surgical patients, and a 6.66-fold increased risk (95\% CI, 1.41-31.47) in emergency or critical patients (Table 1). ${ }^{44)}$

However, the studies also found significant heterogeneity in the instruments used to identify delirium and frailty. These instruments include at least 10 different frailty index or scales (including but not limited to the frailty phenotype, FRAIL Scale, Frailty Index, Clinical Frailty Scale, Edmonton Frail Scale, Erasmus frailty score, Emergency General Surgery Frailty Index, Japanese version of the Cardiovascular Health Study criteria, Groningen Frailty Indicator, mobility impairment, Clinical judgment frailty, Kihon Checklist score, and Comprehensive Geriatric Assessment) and more than five measurement tools for identifying delirium (the Confusion Assessment Method for the Diagnostic and Statistical Manual of Mental Disorders, Fourth Edition; the intensive care delirium screening checklist; the Diagnostic and Statistical Manual of Mental Disorders, Fifth Edition; the Confusion Assessment Method for the intensive care unit, the Delirium Rating Scale Revised-98; and the interRAI-Acute Care for Comprehensive Geriatric Assessment). ${ }^{42,44)}$ Although more studies have examined the interplay between frailty and delirium, historically, studies on this topic have not been sufficient. In their 2017 systematic review, Presico et al. ${ }^{42)}$ identified only 20 studies eligible for systematic review and eight studies for the meta-analysis. Fortunately, more studies have been published since that time; in 2020, Zhang et al. ${ }^{44)}$ identified 30 studies for their meta-analysis. It is promising that the association between frailty and delirium is receiving more attention because the number of patients who may experience these two geriatric conditions is likely to increase along with the rapidly growing number of older adults worldwide. 
Table 1. Recent meta-analysis studies of the association between delirium and frailty

\begin{tabular}{lccl}
\hline Author, year & Number of pooled participants & $\begin{array}{c}\text { Increased risk of delirium } \\
\text { due to frailty }(95 \% \mathrm{CI})\end{array}$ & \multicolumn{1}{c}{ Comments } \\
\hline Presico et al., ${ }^{42)} 2018$ & 999 & $2.19(1.65-2.91)$ & Meta-analysis mainly included observational studies \\
Fu et al., ${ }^{43)} 2021$ & 3,250 & $3.23(2.56-4.09)$ & Meta-analysis only included postoperative patients \\
Zhang et al., $^{42)} 2021$ & 217,623 & $2.96(2.36-3.71)$ & Subgroup analyses of different types of hospitalizations \\
& & -Selective surgical patients (OR=2.43; 95\% CI, 1.88-3.14) \\
& & -Medical patients (OR=3.61; 95\% CI, 1.65-7.89) \\
& & -Urgent surgical patients (OR=3.76; 95\% CI, 2.88-4.92) \\
& & -Emergency or critical illness patients (OR $=6.66 ; 95 \% \mathrm{CI}, 1.41-31.47)$ \\
\hline
\end{tabular}

CI, confidence interval; OR, odds ratio.

\section{ROLE OF THE INTERACTION BETWEEN FRAILTY AND DELIRIUM IN CLINICAL OUTCOMES}

Although it is reassuring that more studies on these two important geriatric syndromes are being conducted, exactly how their interplay results in adverse clinical outcomes and to what extent it affects outcomes remain unknown. As both frailty and delirium are independently associated with adverse clinical outcomes, patients with frailty who develop delirium during hospitalization may experience worse clinical outcomes. However, the existing literature is scarce and is mainly limited to outcomes of mortality or surgical patients as the populations. Furthermore, given the overlapping characteristics between frailty and delirium, frailty may act as a confounder in the association between delirium and clinical outcomes. The inconsistent results from various studies support this hypothesis. Eeles et al. ${ }^{45)}$ assessed the impact of frailty on mortality among older inpatients who had delirium in a single-center study and reported that frail patients had greater long-term mortality among those with delirium compared with that among those without frailty-median survival of 88 days (95\% CI, 5-171) for frail patients vs. 359 days (95\% CI, 118-600) for fit patients. However, Mazzola et al. ${ }^{46)}$ reported that delirium alone was not associated with mortality after adjusting for frailty, supporting the role of frailty as a confounder, increasing the vulnerability to stressors that lead to death. Dani et al. ${ }^{47)}$ conducted a survival analysis to identify the impact of delirium on frailty in hospitalized older adults; interestingly, they reported an inverse gradient of association, with a stronger association observed in the fitter group and a higher hazard ratio for delirium in patients with a lower frailty index.

In addition to mortality as a clinical outcome, Ogawa et al. ${ }^{48)}$ assessed the impact of postoperative frailty on major adverse cardiac events after cardiac surgery in a single-center study. They reported that delirium increased the risk of postoperative frailty $(\mathrm{HR}=2.98$; 95\% CI, 1.46-6.20). Moreover, postoperative frailty increased the risk of a major cardiac adverse event after cardiac surgery $(\mathrm{HR}=$
2.21; 95\% CI, 1.01-4.82). However, after adjusting for delirium, preoperative frailty was not significantly associated with major adverse cardiac events after discharge.

\section{CHALLENGES IN CLINICAL PRACTICE FOR OLDER ADULTS WITH THE COEXISTENCE OF DELIRIUM AND FRAILTY}

Given the high prevalence and complex interactions between the two conditions, managing older adults with both frailty and delirium also becomes challenging. One of the most important challenges in clinical practice is that these two conditions are usually under-recognized, despite their strong associations with adverse clinical outcomes. Hospitalized older adults may experience different types of delirium, including hyperactive, hypoactive, mixed, and without motor symptoms. ${ }^{49}$ However, while hyperactive delirium, mixed-type delirium, or delirium without motor symptoms is easily recognized because of their distinguishing behaviors, hypoactive delirium is less likely to be discovered or reported. ${ }^{49)}$ However, hypoactive delirium may occur more commonly than hyperactive delirium. ${ }^{49-51)}$ Moreover, several studies reported a worse prognosis among patients with hypoactive delirium compared with that among patients with hyperactive delirium. ${ }^{52,53)}$ The under-recognition of delirium may increase its adverse impact on various clinical outcomes. Frailty is also easily missed in clinical practice because there is no standardized systematic screening tool, and some measures are not practical to perform in routine clinical settings. ${ }^{54)}$ For example, Beckert et al. ${ }^{55)}$ reported frailty in a high proportion of patients $(68.8 \%)$ deemed to be surgical candidates and referred for surgeries. They speculated that physicians did not recognize a patient's frailty at the time of referral for surgery. As frailty is a risk factor for delirium, under-recognized frailty among hospitalized older adults can result in a higher incidence of delirium and associated adverse clinical outcomes. Similarly, since delirium may increase the risk of postoperative frailty, the early recogni- 
tion, prevention, and proper management of delirium is also important for providing optimal care for older adults in hospitals.

Another challenge is that as much as they are ignored in routine clinical settings, studies assessing the prevalence of the coexistence of the two conditions or their combined impact on patient outcomes are also scarce. Most studies have focused on the association between these two conditions. Individual estimates for each study with relevant information in the most recent systematic review study by Zhang et al. ${ }^{44)}$ showed a prevalence of the coexistence of the two conditions ranging from $2 \%$ to $26 \%$. Studies with a high prevalence ( $>20 \%$ ) had cohorts with an older age group (mean age, 82.3 or 82.1 years) or included only critically ill patients. Studies with older age groups included patients in either acute general medicine wards ${ }^{45)}$ or an acute geriatric unit. ${ }^{32)}$ However, some studies also showed relatively high prevalence, approximately $15 \%$, in a relatively younger population (mean age, 71 years) and in patients undergoing selective surgery. ${ }^{56)}$ Nevertheless, accurate determination of the association between the prevalence and age or type of admission will require more investigations and systematic reviews. Moreover, very few studies have assessed the impact of the coexistence of the two conditions on patient outcomes. Eeles et al. ${ }^{45)}$ reported that the combination of delirium and frailty was associated with poor survival among patients admitted to general medicine wards. A single-center study by Gandossi et al. ${ }^{57)}$ revealed that the combination of frailty and postoperative delirium was associated with poor functional status upon discharge among hospitalized patients with hip fracture $(\mathrm{RR}=1.47$; 95\% CI, 1.28-1.69). Kwak et al. ${ }^{58)}$ estimated the economic outcomes of various combinations of three geriatric syndromes-delirium, frailty, and dementia-among patients who underwent transcatheter aortic valve replacement procedure and reported that delirium with frailty but without dementia showed the strongest association with higher hospital cost (a 45.1\% increase in hospital cost; 95\% CI, 26.45-66.45).

\section{FUTURE DIRECTIONS}

\section{Identifying High-Risk Patients with Frailty}

The exact pathophysiologic mechanisms of the impact of these two geriatric syndromes on adverse clinical outcomes may be difficult to decipher given their overlapping characteristics. However, previous studies have reported that both frailty and delirium are associated with adverse clinical outcomes; therefore, it is critical to identify these two conditions among hospitalized older adults.

Regardless of whether frailty directly or indirectly increases inpatient mortality, since older adults with frailty are at higher risks for both mortality and delirium, identifying these conditions at an early stage during hospitalization is critical for maximizing patients' clinical outcomes. Subsequently, the American College of Surgeons recommends frailty screening as a part of preoperative assessments, and frailty screening has been tested in various clinical settings. ${ }^{59-61)}$ However, one impediment to such efforts is the lack of consensus on the gold-standard tool for evaluating frailty, as evidenced by the significant heterogeneity in evaluation tools described in previous systematic review or meta-analysis studies. ${ }^{62)}$ Nevertheless, efforts are ongoing to customize frailty assessment tools to accommodate the conditions and specialties of individual clinical settings. ${ }^{44,60,63)}$ Surgery is one specialty that has been actively incorporating frailty assessment in routine preoperative evaluations. ${ }^{64)}$ In orthopedic surgery, Krishnan et al. ${ }^{65)}$ explored the use of a frailty index that was integrated into a comprehensive geriatric assessment for hip fracture patients. More specific frailty assessment tools based on the patient population have also been validated, including the 15-item Trauma-Specific Frailty Index for trauma patients and the 15-variable Emergency General Surgery Specific Frailty Index for patients undergoing emergent surgeries. ${ }^{66,67)}$ In cardiovascular surgery, frailty assessment has become an important part of preoperative assessments to determine if a patient qualifies for less-invasive surgical options such as transcatheter aortic valve replacement rather than surgical aortic valve replacement. ${ }^{68)}$ Additional efforts to facilitate the use of frailty screening in the clinical setting include the incorporation of frailty assessment into the electronic health record system for the convenience of providers and further research on frailty. ${ }^{69)}$

\section{Management of Delirium in Hospitalized Older Adults}

Despite efforts to identify higher-risk patients with frailty, delirium still occurs among hospitalized older adults. The management of delirium in patients with frailty should not differ significantly from the standard management for delirium. The best strategy for preventing delirium among older adults is through multicomponent non-pharmacologic interventions. In their meta-analysis, Hshieh et al. ${ }^{70)}$ showed that a multicomponent non-pharmacologic intervention reduced the incidence of delirium, decreased the length of stay, and improved cost-effectiveness. Moreover, implementing such systematic interventions would reduce the under-recognition of delirium, especially hypoactive delirium, which could be easily missed in routine clinical settings. Indeed, there are several existing models that hospitals could adopt to prevent delirium. Among these, the Hospital Elder Life Program (HELP), developed by Inouye, is one of the most widely used. ${ }^{71-73)}$ The HELP model encourages hospitals to utilize interdisciplinary teams and volunteers to provide practical interventions.

These interventions include reorientation of the patient, promo- 
tion of physical mobilization, therapeutic activities, enhancing nutrition, sleep hygiene, and hearing or visual adaptations. The ABCDEF bundle is another care model that can be used in an intensive care setting. It is a bundled approach to address multiple components of patient care as follows: "A" element: Assess, Prevent, and Manage Pain; "B" element: Both Spontaneous Awakening Trials and Spontaneous Breathing Trials; "C" element: Choice of Analgesia and Sedation; "D” element: Delirium-Assess, Prevent and Manage; "E" element: Early Mobility and Exercise; and "F" element: Family Engagement and Empowerment. ${ }^{74)}$ Such bundled multicomponent approaches that allow healthcare providers to evaluate the components of frailty domains should be beneficial for patients with frailty.

However, the real challenge is that the implementation of such multicomponent interventions requires important resources, which could make the effort non-achievable and non-practical. Alternatively, since the implementation would require an important shift in institutional culture, it may also face barriers from the hospital staff. ${ }^{75)}$ One of the solutions could be the most recent movement in delirium prevention, the Age-Friendly Health System by the Institute for Healthcare Improvement, and the John A. Hartford Foundation, in partnership with the American Hospital Association and the Catholic Health Association of the United States. The Age-Friendly Health System initiative suggests that the healthcare system focus on a set of four evidence-based elements for high-quality care, known as the "4Ms": (1) What Matters-align care strategy with the patient's values or goals; (2) Medicationavoid inappropriate medications; (3) Mentation-recognize delirium, depression, and/or dementia; and (4) Mobility-promote mobility ${ }^{76)}$ Under these four core values for a better quality of care, each healthcare institution can create specific strategies based on its available resources. For example, an institution can choose its own method of assessing mentation (delirium) or mobility (frailty) based on its existing routine practice or documentation tools. In this way, they can incorporate multicomponent interventions to prevent delirium.

As much as multicomponent non-pharmacologic interventions are critical for preventing delirium, the same philosophy regarding multicomponent non-pharmacologic approaches should be applied to the management of delirium. Delirium commonly showed multiple etiologies; thus, the management of delirium should focus on addressing all possible causes of delirium. One strategy is the use of a convenient step-wise approach using the mnemonic DELIRIUM as follows. ${ }^{2)}$ First, the management of delirium should focus on evaluating and treating modifiable contributors to delirium, including Drug, Electrolyte disturbances, Lack of drugs, Infection, Reduced sensory input, Intracranial disorders, Urinary and fecal disorders, and Myocardial and pulmonary disorders. ${ }^{2)}$ Second, the management of delirium should address the prevention and management of any complications such as urinary incontinence, immobility and falls, pressure ulcers, sleep disturbance, and feeding disorders. Then, we should ensure that the patient is comfortable and safe based on the application of behavioral interventions such as de-escalation techniques for agitated patients, family engagement, or pharmacologic interventions, including low-dose antipsychotics, when absolutely necessary. The final step of delirium management is to restore function by optimizing the hospital environment (minimizing clutter and noise and providing adequate lighting and or familiar objects at the bedside), cognitive reconditioning by reorientation, physical therapy, occupational therapy, family support, family participation, and, finally, appropriate discharge planning. ${ }^{2)}$ These approaches allow the early identification of older adults with frailty to identify high-risk patients, prevent delirium, provide proper management of delirium, and prevent further deterioration of physical dysfunction or disability, while simultaneously addressing the components of frailty (Fig. 2).

Although non-pharmacological interventions should be the first-line treatment, there may be cases in which the patient requires pharmacological intervention. Low-dose antipsychotics can be considered in patients who become severely agitated and non-verbal or for whom verbal de-escalating techniques are not effective. However, there has been a debate regarding the use of antipsychotics for hyperactive delirium. Recent systematic reviews have reported different findings. Riviere et al. ${ }^{77)}$ found evidence that quetiapine and olanzapine could be alternatives to haloperidol; however, because of the high levels of heterogeneity among studies, they could not perform a meta-analysis. In contrast, anoth-

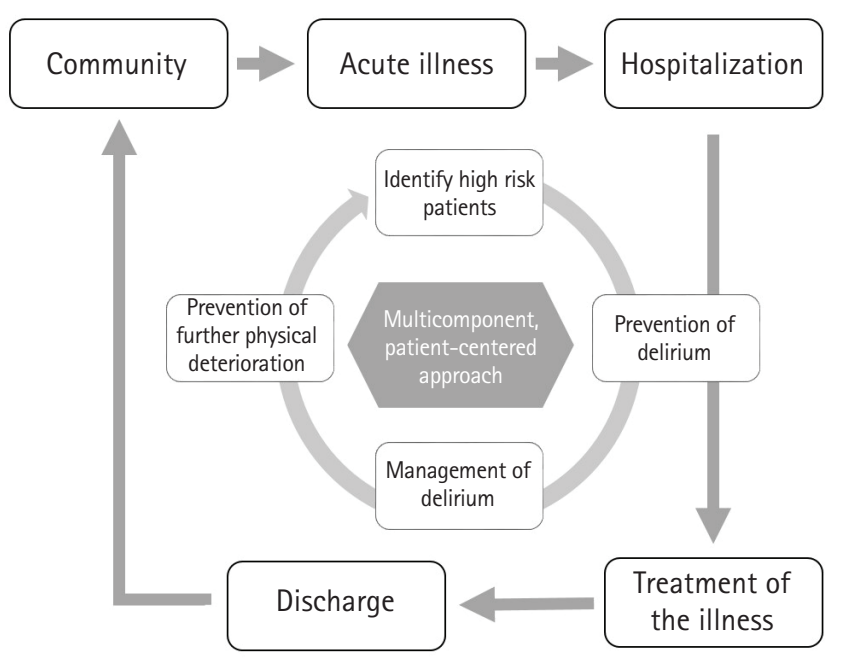

Fig. 2. Integration of a multicomponent patient-centered approach for the prevention and management of delirium with frailty. 
er systematic review by Nikooei et al. ${ }^{78)}$ concluded that there was insufficient evidence to support the routine use of haloperidol or second-generation atypical antipsychotics for the management of delirium. Furthermore, although there is no superior medication that has shown better efficacy, most antipsychotics can cause adverse effects such as prolonged QT interval or extrapyramidal symptoms. Therefore, the choice of antipsychotics is often based on their potential side effects and the patient's specific conditions. ${ }^{2)}$ Nevertheless, specific medication may be indicated in particular cases. For example, benzodiazepine could be first considered for patients with delirium caused by alcohol withdrawal; patients with contraindications to the use of antipsychotics, including neuroleptic malignant syndrome or Parkinson's disease; or patients with dementia with Lewy bodies or seizure disorder. ${ }^{79}$

\section{Geriatric Co-management Model for the Optimal Management of Delirium and Frailty}

As described above, the pathophysiology and management of delirium and frailty require multiple and simultaneous interventions from several different aspects. While these requirements appear holistic and ideal, they are not feasible in busy and highly specialized surgical wards. Therefore, there has been increasing awareness of geriatric co-management and management by both the primary team and geriatricians for high-risk older adults in hospitals. Most studies assessing the effects of geriatric co-management focused on the orthopedic geriatric population. Several studies reported that multidisciplinary geriatric consultation for patients with hip fracture surgery resulted in a reduced incidence of delirium and improved overall quality of care. ${ }^{80-82)}$ Therefore, the idea of geriatric co-management has expanded in surgical oncology. Shahrokni et al. ${ }^{83)}$ conducted a cohort study to assess the impact of geriatric co-management in older adults undergoing surgery for cancer, in which geriatricians provided delirium risk-reduction interventions. However, the main outcome in that study was 90-day postoperative mortality. While the intervention group (geriatric co-management) showed lower 90-day mortality, the authors did not report the impact on delirium incidence. Nevertheless, the geriatric co-management model could be a solution for optimal delirium prevention and management among older adults with physical frailty. Thus, additional studies are warranted.

\section{CONCLUSION}

Delirium and frailty are highly prevalent geriatric syndromes among older adults and are associated with adverse clinical outcomes. While the exact pathophysiology of these two conditions remains unclear, the etiology is multifactorial. Although they are distinct conditions, delirium and frailty share similarities and are significantly associated. Hospitalized older adults with frailty are more likely to develop delirium. Thus, the identification of highrisk patients with frailty is critical for the proper management of delirium. Given the multifactorial etiology of delirium and frailty, the prevention and management of delirium must incorporate a multicomponent patient-centered approach to improve the quality of care among hospitalized older adults.

\section{ACKNOWLEDGMENTS}

\section{CONFLICT OF INTEREST}

The researcher claims no conflicts of interest.

\section{FUNDING}

None.

\section{REFERENCES}

1. American Psychiatric Association. Diagnostic and statistical manual of mental disorders. 5th ed. Washington, DC: American Psychiatric Association; 2013.

2. Marcantonio ER. Delirium in hospitalized older adults. N Engl J Med 2017;377:1456-66.

3. Siriwardhana DD, Hardoon S, Rait G, Weerasinghe MC, Walters KR. Prevalence of frailty and prefrailty among community-dwelling older adults in low-income and middle-income countries: a systematic review and meta-analysis. BMJ Open 2018;8:e018195.

4. O'Caoimh R, Sezgin D, O’Donovan MR, Molloy DW, Clegg A, Rockwood K, et al. Prevalence of frailty in 62 countries across the world: a systematic review and meta-analysis of population-level studies. Age Ageing 2021;50:96-104.

5. Clegg A, Young J, Iliffe S, Rikkert MO, Rockwood K. Frailty in elderly people. Lancet 2013;381:752-62.

6. Gibb K, Seeley A, Quinn T, Siddiqi N, Shenkin S, Rockwood K, et al. The consistent burden in published estimates of delirium occurrence in medical inpatients over four decades: a systematic review and meta-analysis study. Age Ageing 2020;49:352-60.

7. Krewulak KD, Stelfox HT, Leigh JP, Ely EW, Fiest KM. Incidence and prevalence of delirium subtypes in an adult ICU: a systematic review and meta-analysis. Crit Care Med 2018; 46:2029-35.

8. Witlox J, Eurelings LS, de Jonghe JF, Kalisvaart KJ, Eikelenboom $\mathrm{P}$, van Gool WA. Delirium in elderly patients and the risk of postdischarge mortality, institutionalization, and dementia: a meta-analysis. JAMA 2010;304:443-51. 
9. Tieges Z, Quinn T, MacKenzie L, Davis D, Muniz-Terrera G, MacLullich AM, et al. Association between components of the delirium syndrome and outcomes in hospitalised adults: a systematic review and meta-analysis. BMC Geriatr 2021;21:162.

10. Bellelli G, Nobili A, Annoni G, Morandi A, Djade CD, Meagher DJ, et al. Under-detection of delirium and impact of neurocognitive deficits on in-hospital mortality among acute geriatric and medical wards. Eur J Intern Med 2015;26:696-704.

11. Diwell RA, Davis DH, Vickerstaff V, Sampson EL. Key components of the delirium syndrome and mortality: greater impact of acute change and disorganized thinking in a prospective cohort study. BMC Geriatr 2018;18:24.

12. Garcez FB, Jacob-Filho W, Avelino-Silva TJ. Association between level of arousal and 30-day survival in acutely ill older adults. J Am Med Dir Assoc 2020;21:493-9.

13. Inouye SK, Westendorp RG, Saczynski JS. Delirium in elderly people. Lancet 2014;383:911-22.

14. Kwak MJ, Avritscher E, Holmes HM, Jantea R, Flores R, Rianon $\mathrm{N}$, et al. Delirium among hospitalized older adults with acute heart failure exacerbation. J Card Fail 2021;27:453-9.

15. Marengoni A, Zucchelli A, Grande G, Fratiglioni L, Rizzuto D. The impact of delirium on outcomes for older adults hospitalised with COVID-19. Age Ageing 2020;49:923-6.

16. Rebora P, Rozzini R, Bianchetti A, Blangiardo P, Marchegiani A, Piazzoli A, et al. Delirium in patients with SARS-CoV-2 infection: a multicenter study. J Am Geriatr Soc 2021;69:293-9.

17. Davis DH, Muniz Terrera G, Keage H, Rahkonen T, Oinas M, Matthews FE, et al. Delirium is a strong risk factor for dementia in the oldest-old: a population-based cohort study. Brain 2012; 135(Pt9):2809-16.

18. Guerini F, Frisoni GB, Morghen S, Speciale S, Bellelli G, Trabucchi M. Clinical instability as a predictor of negative outcomes among elderly patients admitted to a rehabilitation ward. J Am Med Dir Assoc 2010;11:443-8.

19. Leslie DL, Marcantonio ER, Zhang Y, Leo-Summers L, Inouye SK. One-year health care costs associated with delirium in the elderly population. Arch Intern Med 2008;168:27-32.

20. Maldonado JR. Delirium pathophysiology: an updated hypothesis of the etiology of acute brain failure. Int J Geriatr Psychiatry 2018;33:1428-57.

21. Wilson JE, Mart MF, Cunningham C, Shehabi Y, Girard TD, MacLullich AM, et al. Delirium. Nat Rev Dis Primers 2020;6:90.

22. Shafi MM, Santarnecchi E, Fong TG, Jones RN, Marcantonio ER, Pascual-Leone A, et al. Advancing the neurophysiological understanding of delirium. J Am Geriatr Soc 2017;65:1114-8.

23. Bellelli G, Moresco R, Panina-Bordignon P, Arosio B, Gelfi C, Morandi $\mathrm{A}$, et al. Is delirium the cognitive harbinger of frailty in older adults?: a review about the existing evidence. Front Med (Lausanne) 2017;4:188.

24. Cesari M, Marzetti E, Thiem U, Perez-Zepeda MU, Abellan Van Kan G, Landi F, et al. The geriatric management of frailty as paradigm of "the end of the disease era". Eur J Intern Med 2016;31: 11-4.

25. Morley JE, Vellas B, van Kan GA, Anker SD, Bauer JM, Bernabei R, et al. Frailty consensus: a call to action. J Am Med Dir Assoc 2013;14:392-7.

26. Fried LP, Tangen CM, Walston J, Newman AB, Hirsch C, Gottdiener J, et al. Frailty in older adults: evidence for a phenotype. J Gerontol A Biol Sci Med Sci 2001;56:M146-56.

27. Jones D, Song X, Mitnitski A, Rockwood K. Evaluation of a frailty index based on a comprehensive geriatric assessment in a population based study of elderly Canadians. Aging Clin Exp Res 2005; 17:465-71.

28. Jones DM, Song X, Rockwood K. Operationalizing a frailty index from a standardized comprehensive geriatric assessment. J Am Geriatr Soc 2004;52:1929-33.

29. Evans SJ, Sayers M, Mitnitski A, Rockwood K. The risk of adverse outcomes in hospitalized older patients in relation to a frailty index based on a comprehensive geriatric assessment. Age Ageing 2014;43:127-32.

30. Rockwood K, Mitnitski A. Frailty in relation to the accumulation of deficits. J Gerontol A Biol Sci Med Sci 2007;62:722-7.

31. Clegg A, Young J. The frailty syndrome. Clin Med (Lond) 2011; 11:72-5.

32. Bellelli PG, Biotto M, Morandi A, Meagher D, Cesari M, Mazzola $\mathrm{P}$, et al. The relationship among frailty, delirium and attentional tests to detect delirium: a cohort study. Eur J Intern Med 2019; 70:33-8.

33. Quinlan N, Marcantonio ER, Inouye SK, Gill TM, Kamholz B, Rudolph JL. Vulnerability: the crossroads of frailty and delirium. J Am Geriatr Soc 2011;59(Suppl 2):S262-8.

34. Giunta S. Exploring the complex relations between inflammation and aging (inflamm-aging): anti-inflamm-aging remodelling of inflamm- aging, from robustness to frailty. Inflamm Res 2008; 57:558-63.

35. Leng SX, Xue QL, Tian J, Walston JD, Fried LP. Inflammation and frailty in older women. J Am Geriatr Soc 2007;55:864-71.

36. Kuo HK, Lipsitz LA. Cerebral white matter changes and geriatric syndromes: is there a link? J Gerontol A Biol Sci Med Sci 2004; 59:818-26.

37. Klein BE, Klein R, Knudtson MD, Lee KE. Frailty, morbidity and survival. Arch Gerontol Geriatr 2005;41:141-9.

38. Newman AB, Gottdiener JS, Mcburnie MA, Hirsch CH, Kop WJ, Tracy R, et al. Associations of subclinical cardiovascular dis- 
ease with frailty. J Gerontol A Biol Sci Med Sci 2001;56:M15866.

39. Rudolph JL, Marcantonio ER. Review articles: postoperative delirium: acute change with long-term implications. Anesth Analg 2011;112:1202-11.

40. Lesourd B. Nutrition: a major factor influencing immunity in the elderly. J Nutr Health Aging 2004;8:28-37.

41.Inouye SK. Delirium in older persons. N Engl J Med 2006; 354:1157-65.

42. Persico I, Cesari M, Morandi A, Haas J, Mazzola P, Zambon A, et al. Frailty and delirium in older adults: a systematic review and meta-analysis of the literature. J Am Geriatr Soc 2018;66:202230.

43. Fu D, Tan X, Zhang M, Chen L, Yang J. Association between frailty and postoperative delirium: a meta-analysis of cohort study. Aging Clin Exp Res 2021 Apr 8 [Epub]. https://doi. org/10.1007/s40520-021-01828-9.

44. Zhang XM, Jiao J, Xie XH, Wu XJ. The association between frailty and delirium among hospitalized patients: an updated meta-analysis. J Am Med Dir Assoc 2021;22:527-34.

45. Eeles EM, White SV, O’Mahony SM, Bayer AJ, Hubbard RE. The impact of frailty and delirium on mortality in older inpatients. Age Ageing 2012;41:412-6.

46. Mazzola P, Tassistro E, Di Santo S, Rossi E, Andreano A, Valsecchi MG, et al. The relationship between frailty and delirium: insights from the 2017 Delirium Day study. Age Ageing 2021 Mar 31 [Epub]. https://doi.org/10.1093/ageing/afab042.

47. Dani M, Owen LH, Jackson TA, Rockwood K, Sampson EL, Davis D. Delirium, frailty, and mortality: interactions in a prospective study of hospitalized older people. J Gerontol A Biol Sci Med Sci 2018;73:415-8.

48. Ogawa M, Izawa KP, Satomi-Kobayashi S, Tsuboi Y, Komaki K, Gotake Y, et al. Impact of delirium on postoperative frailty and long term cardiovascular events after cardiac surgery. PLoS One 2017; 12:e0190359.

49. Albrecht JS, Marcantonio ER, Roffey DM, Orwig D, Magaziner J, Terrin M, et al. Stability of postoperative delirium psychomotor subtypes in individuals with hip fracture. J Am Geriatr Soc 2015;63:970-6.

50. Boettger S, Breitbart W. Phenomenology of the subtypes of delirium: phenomenological differences between hyperactive and hypoactive delirium. Palliat Support Care 2011;9:129-35.

51. Meagher DJ, Leonard M, Donnelly S, Conroy M, Adamis D, Trzepacz PT. A longitudinal study of motor subtypes in delirium: frequency and stability during episodes. J Psychosom Res 2012;72:236-41.

52. Meagher DJ, Leonard M, Donnelly S, Conroy M, Adamis D,
Trzepacz PT. A longitudinal study of motor subtypes in delirium: relationship with other phenomenology, etiology, medication exposure and prognosis. J Psychosom Res 2011;71:395403.

53. Robinson TN, Raeburn CD, Tran ZV, Brenner LA, Moss M. Motor subtypes of postoperative delirium in older adults. Arch Surg 2011;146:295-300.

54. Martin FC, Brighton P. Frailty: different tools for different purposes? Age Ageing 2008;37:129-31.

55. Beckert AK, Huisingh-Scheetz M, Thompson K, Celauro AD, Williams J, Pachwicewicz P, et al. Screening for frailty in thoracic surgical patients. Ann Thorac Surg 2017;103:956-61.

56. Jung P, Pereira MA, Hiebert B, Song X, Rockwood K, Tangri N, et al. The impact of frailty on postoperative delirium in cardiac surgery patients. J Thorac Cardiovasc Surg 2015;149:869-75.

57. Gandossi CM, Zambon A, Oliveri G, Codognola M, Szabo H, Cazzulani I, et al. Frailty, post-operative delirium and functional status at discharge in patients with hip fracture. Int J Geriatr Psychiatry 2021 Apr 27 [Epub]. https://doi.org/10.1002/gps. 5561.

58. Kwak MJ, Rasu R, Morgan RO, Lee J, Rianon NJ, Holmes HM, et al. The association of economic outcome and geriatric syndromes among older adults with transcatheter aortic valve replacement (TAVR). J Health Econ Outcomes Res 2020;7:17581.

59. Chow WB, Rosenthal RA, Merkow RP, Ko CY, Esnaola NF; American College of Surgeons National Surgical Quality Improvement Program, et al. Optimal preoperative assessment of the geriatric surgical patient: a best practices guideline from the American College of Surgeons National Surgical Quality Improvement Program and the American Geriatrics Society. J Am Coll Surg 2012;215:453-66.

60. Afilalo J, Alexander KP, Mack MJ, Maurer MS, Green P, Allen LA, et al. Frailty assessment in the cardiovascular care of older adults. J Am Coll Cardiol 2014;63:747-62.

61. van Dam CS, Hoogendijk EO, Mooijaart SP, Smulders YM, de Vet RC, Lucke JA, et al. A narrative review of frailty assessment in older patients at the emergency department. EurJ Emerg Med 2021;28:266-76.

62. Walston J, Bandeen-Roche K, Buta B, Bergman H, Gill TM, Morley JE, et al. Moving frailty toward clinical practice: NIA Intramural Frailty Science Symposium summary. J Am Geriatr Soc 2019;67:1559-64.

63. Lee H, Lee E, Jang IY. Frailty and comprehensive geriatric assessment. J Korean Med Sci 2020;35:e16.

64. Poh AWY, Teo SP. Utility of frailty screening tools in older surgical patients. Ann Geriatr Med Res 2020;24:75-82. 
65. Krishnan M, Beck S, Havelock W, Eeles E, Hubbard RE, Johansen A. Predicting outcome after hip fracture: using a frailty index to integrate comprehensive geriatric assessment results. Age Ageing 2014;43:122-6.

66. Joseph B, Pandit V, Zangbar B, Kulvatunyou N, Tang A, O’Keeffe $\mathrm{T}$, et al. Validating trauma-specific frailty index for geriatric trauma patients: a prospective analysis. J Am Coll Surg 2014;219:107.

67. Orouji Jokar T, Ibraheem K, Rhee P, Kulavatunyou N, Haider A, Phelan HA, et al. Emergency general surgery specific frailty index: a validation study. J Trauma Acute Care Surg 2016;81:25460.

68. Mack MJ, Leon MB, Thourani VH, Makkar R, Kodali SK, Russo $\mathrm{M}$, et al. Transcatheter aortic-valve replacement with a balloon-expandable valve in low-risk patients. N Engl J Med 2019;380:1695-705.

69. Kim DH. Measuring frailty in health care databases for clinical care and research. Ann Geriatr Med Res 2020;24:62-74.

70. Hshieh TT, Yue J, Oh E, Puelle M, Dowal S, Travison T, et al. Effectiveness of multicomponent nonpharmacological delirium interventions: a meta-analysis. JAMA Intern Med 2015;175: 512-20.

71. Inouye SK, Bogardus ST Jr, Charpentier PA, Leo-Summers L, Acampora D, Holford TR, et al. A multicomponent intervention to prevent delirium in hospitalized older patients. N Engl J Med 1999;340:669-76.

72. Inouye SK. Prevention of delirium in hospitalized older patients: risk factors and targeted intervention strategies. Ann Med 2000;32:257-63.

73. Inouye SK, Bogardus ST Jr, Baker DI, Leo-Summers L, Cooney LM Jr. The Hospital Elder Life Program: a model of care to prevent cognitive and functional decline in older hospitalized patients. Hospital Elder Life Program. J Am Geriatr Soc 2000; 48:1697-706.

74. Ely EW. The ABCDEF bundle: science and philosophy of how
ICU liberation serves patients and families. Crit Care Med 2017;45:321-30.

75. Greysen SR. Delirium and the "know-do" gap in acute care for elders. JAMA Intern Med 2015;175:521-2.

76. Institute for Healthcare Improvement. Age Friendly Health Systems: what is an age-friendly health system? [Internet]. Cambridge, MA: Institute for Healthcare Improvement; c2021 [cited 2021 Aug 3]. Available from: http://www.ihi.org/Engage/Initiatives/Age-Friendly-Health-Systems/Pages/default.aspx.

77. Riviere J, van der Mast RC, Vandenberghe J, Van Den Eede F. Efficacy and tolerability of atypical antipsychotics in the treatment of delirium: a systematic review of the literature. Psychosomatics 2019;60:18-26.

78. Nikooie R, Neufeld KJ, Oh ES, Wilson LM, Zhang A, Robinson KA, et al. Antipsychotics for treating delirium in hospitalized adults: a systematic review. Ann Intern Med 2019;171:485-95.

79. Bellelli G, Brathwaite JS, Mazzola P. Delirium: a marker of vulnerability in older people. Front Aging Neurosci 2021;13: 626127.

80. Deschodt M, Braes T, Flamaing J, Detroyer E, Broos P, Haentjens $\mathrm{P}$, et al. Preventing delirium in older adults with recent hip fracture through multidisciplinary geriatric consultation. J Am Geriatr Soc 2012;60:733-9.

81. Marcantonio ER, Flacker JM, Wright RJ, Resnick NM. Reducing delirium after hip fracture: a randomized trial. J Am Geriatr Soc 2001;49:516-22.

82. Fisher AA, Davis MW, Rubenach SE, Sivakumaran S, Smith PN, Budge MM. Outcomes for older patients with hip fractures: the impact of orthopedic and geriatric medicine cocare. J Orthop Trauma 2006;20:172-80.

83. Shahrokni A, Tin AL, Sarraf S, Alexander K, Sun S, Kim SJ, et al. Association of geriatric comanagement and 90-day postoperative mortality among patients aged 75 years and older with cancer.JAMA Netw Open 2020;3:e209265. 